\title{
Localization of Primitives Using Adaptive Projections
}

\author{
Yaacov Hel-Or * Amir Shmuel Michael Werman \\ Department of Computer Science \\ The Hebrew University of Jerusalem \\ 91904 Jerusalem \\ Israel
}

\begin{abstract}
An application of an active method in order to compute highly accurate 3D localization of point features from few projections is presented. The angle of projection of the image is controlled by the system and directed to extract 3D information from the environment in a manner leading to accurate location in less computation.

This model is relevant for tomographic reconstruction, for feature based stereo and for model based robot registration.
\end{abstract}

\section{Introduction}

Image analysis embraces a wide range of image processing tasks, including object localization and identification. Traditional approachs to these tasks have entailed a passive approach to the data collection problem, the main emphasis being on the algorithms to extract the exact information from the images already taken. The fullfillment of computer vision tasks is based on analysis of a given image or image sequence. The recognition process has no control over the imaging process. The results usually have no reliability measure associated with them. Consequently, current systems are often characterized by such shortcomings as problems with uniqueness, accuracy and/or stability.

An active approach to the imaging process is advocated, in order to achieve more robust and reliable results using less computation. Active vision, as referred to in this paper, is input dependent data acquisition, coupled with a treatment of the reliability of the acquired information. A mathematical model is proposed that includes the known information in the scene and the expected information to be sensed by the system. From the model a new optimal sensing position is determined. A picture taken from this new position should enable the system to deduce as much new 3D information as possible. Generally, the 3D information is composed of location in space and its certainty. Intelligent active perception includes algorithms for updating the known information according to the input, positioning the sensor and judging the reliability of the information that the

${ }^{*}$ Y.H. was supported by the Leibniz Center for Reseach in Computer Science. 
system already has. Kalman filtering techniques [5] are used for updating information, as Kalman filtering is an optimal method of integrating data.

The proposed approach contributes to the following problems: improving the stability of solutions and the ability to perform tasks in a noisy environment; improving exactness and reliability of solutions; and reducing the quantity of data needed and the time for its analysis. Reducing the amount of necessary data and speeding its analysis is a side effect of gathering only the information needed to complete the computation, rather than collecting information in a random manner.

There has been some previous work done using active vision as a model for robot vision using different aspects of vision such as vergence, stereo, fixation and focus in a human like manner. These papers treat the control, activation and reinforcement of certain of the sensing modes on the others $[4,3,1]$.

This paper describes an application of the active method in order to compute highly accurate 3D localization of point features from few projections. The angle of projection of the image is controlled by the system and directed to extract 3D information from the environment in a manner leading to accurate location on less computation. After each phase of computation (between projections), the information already computed is assessed and when information is still needed a new optimal angle for the next projection is derived. This process repeatedly takes place, until a sufficient accuracy is achieved in the solution.

This model is relevant for tomographic reconstraction and for robot registration based on scene model.

Section 2 contains an introduction of the Gauss-Markov model and the Kalman filter. Sections 3 and 4 treat the 2 and 3D cases in detail. The imaging model and the computation of optimal angle for the next projection are described. Simulation results are given in section 5 .

\section{The Static Gauss-Markov Model and the Kalman Filter}

The Static Gauss-Markov discrete time model is a particular case of the general GaussMarkov Model and describes a class of physical or abstract systems. The state of the system is described by a finite dimensional vector $s$. This state is measured at time $t$ by $m_{t}$ which is the result of a linear transformation $\left(H_{t}^{T}\right)$ of the state contaminated by an additive noise process $v_{t}$ :

$$
\begin{aligned}
m_{t} & =H_{t}^{T} s+v_{t} \\
v_{t} & \sim N\left(0, R_{t}\right) .
\end{aligned}
$$

(The subscript $t$ denotes a time argument).

The noise process is described as a zero mean Gaussian process, with known covariance matrix $R_{t}$. It is characterized as a white process in the sense that for any two different time instants $k$ and $l, v_{k}$ and $v_{l}$ are independent random variables.

For the model described above, the static Kalman filter [5, 2] is a method for optimally estimating the system state by using the measurements recorded up to the current time. 
Current estimation of the system state $\hat{s}_{t}$ is in effect the conditional expectance

$$
\hat{s}_{t}=E\left(s \mid m_{1}, m_{2}, \ldots, m_{t}\right) \quad \text { for } t>0
$$

( $\hat{X}$ for any $X$ means the estimated $X$ ).

The quality of this estimate is given by the error covariance matrix

$$
\Sigma_{t}=E\left(\left[s-\hat{s}_{t}\right]\left[s-\hat{s}_{t}\right]^{T} \mid m_{1}, m_{2}, \ldots, m_{t}\right) \quad \text { for } t>0 .
$$

The Kalman filter is an unbiased estimator, and in addition to that it is optimal with respect to the minimum variance criteria;

$$
\operatorname{trace} \Sigma_{t}=E\left(\left\|s-\hat{s}_{t}\right\|^{2} \mid m_{1}, m_{2}, \ldots, m_{t}\right) \quad \text { for } t>0
$$

is the conditional error variance associated with the estimate $\hat{s}_{t}$, and $\hat{s}_{t}=E\left(s_{t} \mid m_{1}, m_{2}, \ldots, m_{t}\right)$ minimizes this error variance.

The explicit state update and error covariance matrix update euations, describing one step of the static Kalman filter, are:

$$
\begin{aligned}
& \hat{s}_{t+1}=\hat{s}_{t}+\Sigma_{t} H_{t+1}\left(H_{t+1}^{T} \Sigma_{t} H_{t+1}+R_{t+1}\right)^{-1}\left(m_{t+1}-H_{t+1}^{T} \hat{s}_{t}\right) \\
& \Sigma_{t+1}=\Sigma_{t}-\Sigma_{t} H_{t+1}\left(H_{t+1}^{T} \Sigma_{t} H_{t+1}+R_{t+1}\right)^{-1} H_{t+1}^{T} \Sigma_{t}
\end{aligned}
$$

$\hat{s}_{0}$ and $\Sigma_{0}$ are assumed to be known due to prior knowledge.

The incremental character of the equations enables the extraction of all the information realized by previous measurements by using the estimation and variance corresponding to the current time.

Further description of both the Gauss-Markov model and the Kalman filter can be found in $[2,5]$.

\section{Uncertainty Driven Active Projection}

\subsection{Optimal Positioning of a Single Feature}

The model described through this section is concerned with the 2D plane specified by the Cartesian coordinates $(X, Y)$. Let $F$ be a feature point in the 2D plane. The position of $F\left(P_{F}\right)$ is uncertain, and modelled in time $t$ as a bivariate Gaussian random vector

$$
P_{F}=\left(\begin{array}{c}
X_{F} \\
Y_{F}
\end{array}\right) \sim N\left(\left(\hat{P}_{F}\right)_{t},\left(\Sigma_{F}\right)_{t}\right)
$$

where

$$
\left(\hat{P}_{F}\right)_{t}=\left(\begin{array}{c}
\hat{X}_{F} \\
\hat{Y}_{F}
\end{array}\right)_{t} \quad \text { and } \quad\left(\Sigma_{F}\right)_{t}=\left(\begin{array}{cc}
\sigma_{x x}^{2} & \sigma_{x y}^{2} \\
\sigma_{x y}^{2} & \sigma_{y y}^{2}
\end{array}\right)_{t}
$$

are the current estimation of the position of $F$ and the covariance matrix that evaluates this estimation. 


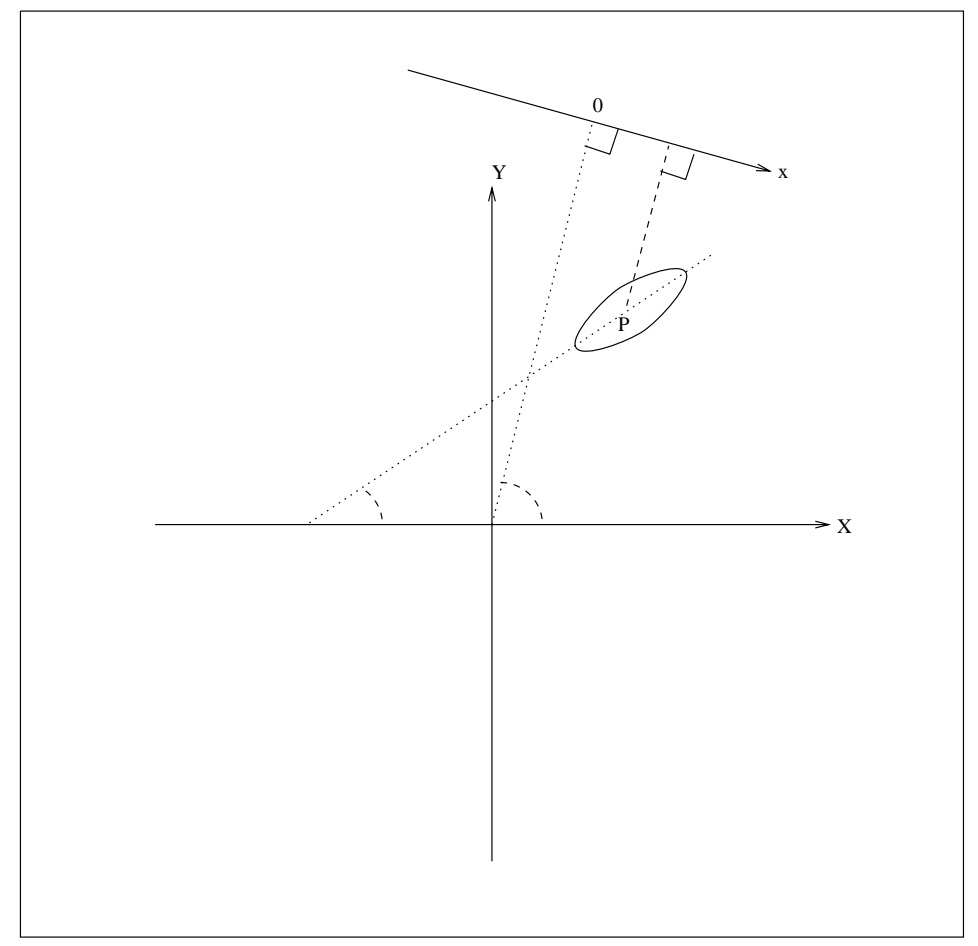

Figure 1: Coordinate systems of the 2D plane and measurement projection line. $\theta$ is the projection angle and $\alpha$ is the angle by which the axes are rotated.

Naturally, reduction of the uncertainty of the feature location is needed. The means by which this reduction takes place is a sequence of orthographic projections of $P_{F}$ onto a line $\ell$ residing in the plane (see Figure 1). For each projection, the line may be rotated differently relative to the axes. Locations on $\ell$ are specified by their coordinate $x$ on the line. Any measurement $x_{t}$ that takes place in time $t$ is corrupted by an additive zero mean Gaussian random noise $v_{t}$, whose variance $R_{t}$ is known.

In order to refine the estimation of the position of $F$, the static Kalman filter is used. The state vector corresponds to the coordinates of the feature:

$$
s_{F}=P_{F}=\left(\begin{array}{c}
X_{F} \\
Y_{F}
\end{array}\right) \text {. }
$$

Suppose that the measurement projection line $\ell$ is rotated $\theta_{t}^{\circ}$ relative to the $X$ axis in time $t$. Thus, the transformation matrix specifying the measurement is

$$
H_{t}^{T}=\left(-\sin \theta_{t}, \cos \theta_{t}\right) .
$$

The appropriate static Gauss-Markov measurement equation is

$$
m_{t}=\left(x_{F}\right)_{t}=H_{t}^{T} P_{F}+v_{t}
$$

Substituting $\left(\hat{P}_{F}\right)_{t}, H_{t}$ and $\left(\Sigma_{F}\right)_{t}$ in the static Kalman filter updating equations (Eq. 2) results by two expressions specifying the values of $\left(\Sigma_{F}\right)_{t+1}$ and $\left(\hat{P}_{F}\right)_{t+1}$ :

$$
\begin{aligned}
\left(\hat{P}_{F}\right)_{t+1} & =\left(\hat{P}_{F}\right)_{t}+\left(\Sigma_{F}\right)_{t} H_{t+1}\left[H_{t+1}^{T}\left(\Sigma_{F}\right)_{t} H_{t+1}+R_{t+1}\right]^{-1}\left[\left(x_{F}\right)_{t+1}-H_{t+1}^{T}\left(\hat{P}_{F}\right)_{t}\right] \\
\left(\Sigma_{F}\right)_{t+1} & =\left(\Sigma_{F}\right)_{t}-\left(\Sigma_{F}\right)_{t} H_{t+1}\left[H_{t+1}^{T}\left(\Sigma_{F}\right)_{t} H_{t+1}+R_{t+1}\right]^{-1} H_{t+1}^{T}\left(\Sigma_{F}\right)_{t}
\end{aligned}
$$


Recall that $\operatorname{trace}\left(\Sigma_{t}\right)$ is the conditional error variance associated with the estimate $\hat{s}_{t}$ of the static Kalman filter. To optimally estimate $P_{F}$ and accelerate the reduction of uncertainty resulting by the next measurement, $\operatorname{trace}\left(\Sigma_{F}\right)_{t+1}$ should be minimized. The trace operator is invariant under axis rotation. For the simplicity of demonstration, the axes are rotated in order to diagonalize $\Sigma_{t}$ resulting in $\Sigma_{t}^{\prime}$ (prime denotes the value in the new coordinate system). A cross section parallel to the $X Y$ plane cutting the Gaussian represented by $\Sigma_{t}^{\prime}$ is an ellipse whose axes are parallel to the $X, Y$ axes.

Rotation of the axes by an angle $\alpha$ where

$$
\alpha=\frac{1}{2} \cdot \arctan \left(\frac{2 \sigma_{x y}^{2}}{\sigma_{y y}^{2}-\sigma_{x x}^{2}}\right)
$$

gives

$$
\Sigma_{t}^{\prime}=\left(\begin{array}{cc}
\sigma_{x x}^{\prime 2} & 0 \\
0 & \sigma_{y y}^{\prime 2}
\end{array}\right) \text { and } H_{t}^{\prime}=\left(-\sin \left(\theta_{t}-\alpha\right), \cos \left(\theta_{t}-\alpha\right)\right)
$$

To determine $\alpha$ uniquely, the condition $\sigma_{x x}^{\prime 2} \geq \sigma_{y y}^{\prime 2}$ is imposed (i.e. the major axis of the ellipse is parallel to the $x$ axis). The corresponding values of $\sigma_{x x}^{\prime 2}$ and $\sigma_{y y}^{\prime 2}$ are

$$
\sigma_{x x}^{\prime 2}=\frac{1}{2}\left(\sigma_{x x}^{2}+\sigma_{y y}^{2}\right)+\sqrt{\frac{1}{4}\left(\sigma_{x x}^{2}-\sigma_{y y}^{2}\right)^{2}+\sigma_{x y}^{4}}
$$

and

$$
\sigma_{y y}^{\prime 2}=\frac{1}{2}\left(\sigma_{x x}^{2}+\sigma_{y y}^{2}\right)-\sqrt{\frac{1}{4}\left(\sigma_{x x}^{2}-\sigma_{y y}^{2}\right)^{2}+\sigma_{x y}^{4}}
$$

Substituting $H_{t}$ by $H_{t}^{\prime}$ and $\Sigma_{t}$ by $\Sigma_{t}^{\prime}$ in Eq. 3 results in

$$
\Sigma_{t+1}^{\prime}=\frac{1}{\sigma_{x x}^{\prime 2} \sin ^{2} \theta^{\prime}+\sigma_{y y}^{\prime 2} \cos ^{2} \theta^{\prime}+R_{t}}\left(\begin{array}{cc}
\sigma_{x x}^{\prime 2} \sigma_{y y}^{\prime 2} \cos ^{2} \theta^{\prime}+\sigma_{x x}^{\prime 2} R_{t} & \sigma_{x x}^{\prime 2} \sigma_{y y}^{\prime 2} \sin \theta^{\prime} \cos \theta^{\prime} \\
\sigma_{x x}^{\prime 2} \sigma_{y y}^{\prime 2} \sin \theta^{\prime} \cos \theta^{\prime} & \sigma_{x x}^{\prime 2} \sigma_{y y}^{\prime 2} \sin ^{2} \theta^{\prime}+\sigma_{y y}^{\prime 2} R_{t}
\end{array}\right)
$$

and

$$
\operatorname{trace}\left(\Sigma_{t+1}^{\prime}\right)=\operatorname{trace}\left(\Sigma_{t+1}\right)=\frac{\sigma_{x x}^{\prime 2} \sigma_{y y}^{\prime 2}+R_{t}\left(\sigma_{x x}^{\prime 2}+\sigma_{y y}^{\prime 2}\right)}{\sigma_{x x}^{\prime 2} \sin ^{2} \theta^{\prime}+\sigma_{y y}^{\prime 2} \cos ^{2} \theta^{\prime}+R_{t}}
$$

where

$$
\theta_{t}^{\prime}=\theta_{t}-\alpha
$$

Taking the derivative of Equation (4) with respect to $\theta$ shows that the minimum of $\operatorname{trace}\left(\Sigma_{F}\right)_{t+1}$ is obtained for

$$
\begin{aligned}
& \theta_{\min 1}=\frac{\pi}{2}+\alpha \\
& \theta_{\min 2}=\frac{3 \pi}{2}+\alpha
\end{aligned}
$$

The two different results are actually equal, since the results of projections in the two different directions differ only by the sign. The result is in accordance with our intuition since it prefers the projection to be performed on a line which is parallel to the major axis of the ellipse. The information about the location of $F$ along this direction is the most inexact compared to the other directions. Thus, measuring in the direction $\theta_{\min 1}$ or $\theta_{\min 2}$ reduces the uncertainty in positioning along the "worst" direction, resulting by the largest possible reduction of the global uncertainty. 


\subsection{Optimal Positioning of Some Features}

Most application require the positioning of more than one feature at a time. The following subsection includes an extension of the previous subsection result to handle the case of $n$ features in the plane.

The appropriate state vector is

$$
s=\left(\begin{array}{c}
P_{1} \\
P_{2} \\
\vdots \\
P_{n}
\end{array}\right), \quad \text { where } \quad P_{i}=\left(\begin{array}{c}
X_{i} \\
Y_{i}
\end{array}\right)
$$

is the position of the $i^{\text {th }}$ feature.

The uncertainty corresponding to the estimation of the position of the $i^{\text {th }}$ feature in time $t$ is represented by the covariance matrix

$$
\left(\Sigma_{i}\right)_{t}=\left(\begin{array}{ll}
\left(\sigma_{x x}^{2}\right)_{i} & \left(\sigma_{x y}^{2}\right)_{i} \\
\left(\sigma_{x y}^{2}\right)_{i} & \left(\sigma_{y y}^{2}\right)_{i}
\end{array}\right)_{t} .
$$

Assuming that no correlation exists among the locations of different features, the global covariance matrix is

$$
\Sigma_{t}=\left(\begin{array}{cccc}
\left(\Sigma_{1}\right)_{t} & & & 0 \\
& \left(\Sigma_{2}\right)_{t} & & \\
0 & & \ddots & \\
0 & & & \left(\Sigma_{n}\right)_{t}
\end{array}\right)
$$

The measurement vector $m_{t}$ represents the projections in time $t$ of the $n$ features on the line $\ell$ rotated by $\theta_{t}$ radians relative to the $X$ axis. Thus the transformation matrix specifying the measurement is

$$
H_{t}=\left(\begin{array}{cccc}
\left(H_{1}\right)_{t} & & & 0 \\
& \left(H_{2}\right)_{t} & & \\
& & \ddots & \\
0 & & & \left(H_{n}\right)_{t}
\end{array}\right) \quad \text { where } \quad\left(H_{i}\right)_{t}^{T}=\left(-\sin \theta_{t}, \cos \theta_{t}\right)
$$

The equation specifying the measurement (Eq. 1) still holds:

$$
m_{t}=\left(\begin{array}{c}
m_{1} \\
m_{2} \\
\vdots \\
m_{n}
\end{array}\right)_{t}=\left(\begin{array}{c}
x_{1} \\
x_{2} \\
\vdots \\
x_{n}
\end{array}\right)_{t}=H_{t}^{T} s+v_{t}
$$

where

$$
v_{t}=\left(\begin{array}{c}
v_{1} \\
v_{2} \\
\vdots \\
v_{n}
\end{array}\right)_{t} \quad \text { and } \quad\left(v_{i}\right)_{t} \sim N\left(0,\left(R_{i}\right)_{t}\right)
$$


Substituting the above mentioned values into Equation (2) gives

$$
\Sigma_{t+1}=\left(\begin{array}{cccc}
\left(\Sigma_{1}\right)_{t+1} & & & 0 \\
& \left(\Sigma_{2}\right)_{t+1} & & \\
0 & & \ddots & \\
0 & & & \left(\Sigma_{n}\right)_{t+1}
\end{array}\right)
$$

where for each $i\left(\Sigma_{i}\right)_{t+1}$ is derived by Equation (3).

Thus

$$
\operatorname{trace}\left(\Sigma_{t+1}\right)=\sum_{i=1}^{n} \operatorname{trace}\left(\left(\Sigma_{i}\right)_{t+1}\right)
$$

Using the value corresponding to $\operatorname{trace}\left(\Sigma_{i}\right)_{t+1}$ (Equation 4$)$ we get

$$
\operatorname{trace}\left(\Sigma_{t+1}\right)=\sum_{i=1}^{n} \frac{\left(\sigma_{x x}^{\prime 2}\right)_{i}\left(\sigma_{y y}^{\prime 2}\right)_{i}+\left(R_{i}\right)_{t}\left[\left(\sigma_{x x}^{\prime 2}\right)_{i}+\left(\sigma_{y y}^{\prime 2}\right)_{i}\right]}{\left(\sigma_{x x}^{\prime 2}\right)_{i} \sin ^{2} \theta_{i}^{\prime}+\left(\sigma_{y y}^{\prime 2}\right)_{i} \cos ^{2} \theta_{i}^{\prime}+\left(R_{i}\right)_{t}}
$$

where

$$
\begin{gathered}
\theta_{i}^{\prime}=\theta-\alpha_{i} \\
\alpha_{i}=\frac{1}{2} \arctan \left(\frac{2\left(\sigma_{x y}^{2}\right)_{i}}{\left(\sigma_{y y}^{2}\right)_{i}-\left(\sigma_{x x}^{2}\right)_{i}}\right) \\
\left(\sigma_{x x}^{\prime 2}\right)_{i}=\frac{1}{2}\left[\left(\sigma_{x x}^{2}\right)_{i}+\left(\sigma_{y y}^{2}\right)_{i}\right]+\sqrt{\frac{1}{4}\left[\left(\sigma_{x x}^{2}\right)_{i}-\left(\sigma_{y y}^{2}\right)_{i}\right]^{2}+\left(\sigma_{x y}^{2}\right)_{i}^{2}}
\end{gathered}
$$

and

$$
\left(\sigma_{y y}^{\prime 2}\right)_{i}=\frac{1}{2}\left[\left(\sigma_{x x}^{2}\right)_{i}+\left(\sigma_{y y}^{2}\right)_{i}\right]-\sqrt{\frac{1}{4}\left[\left(\sigma_{x x}^{2}\right)_{i}-\left(\sigma_{y y}^{2}\right)_{i}\right]^{2}+\left(\sigma_{x y}^{2}\right)_{i}^{2}} .
$$

Let

$$
\xi_{i}=\left(\sigma_{x x}^{\prime 2}\right)_{i}\left(\sigma_{y y}^{\prime 2}\right)_{i}+\left(R_{i}\right)_{t}\left[\left(\sigma_{x x}^{\prime 2}\right)_{i}+\left(\sigma_{y y}^{\prime 2}\right)_{i}\right]
$$

for each $i, \xi_{i}$ is known and constant. Equation (5) can be rewritten by

$$
\operatorname{trace}\left(\Sigma_{t+1}\right)=\sum_{i=1}^{n} \frac{\xi_{i}}{\left(\sigma_{x x}^{\prime 2}\right)_{i} \sin ^{2} \theta_{i}^{\prime}+\left(\sigma_{y y}^{\prime 2}\right)_{i} \cos ^{2} \theta_{i}^{\prime}+\left(R_{i}\right)_{t}}
$$

The direction $\theta_{\text {min }}$ of the projection in time $t$ which minimizes $\operatorname{trace}\left(\Sigma_{t+1}\right)$ is the direction which maximally reduces the global uncertainty of the features estimated positioning:

$$
\theta_{\min }=\min _{\theta}\left[\operatorname{trace}\left(\Sigma_{t+1}\right)\right]
$$

For each individual feature $i, \operatorname{trace}\left(\Sigma_{i}\right)_{t+1}$ has two minima as was previously shown. However, $\operatorname{trace}\left(\Sigma_{t+1}\right)$ has up to $2 n$ local minima. Using simple iterative methods we can find a local minimum which is close to the initial state. To find the global minimum one must 
start the iterative process at a point close to its location. In our case the measurement noise $\left(R_{i}\right)_{t}$ can be artificially increased in trace $\left(\Sigma_{t+1}\right)$ so that only a single minimum is left. This minimum is close to $\theta_{\min }$ and thus it can be used as the initial state in the iterative process. The iterative process should now incorporate decreasing the measurement noise to its real values, so as to converge to the real global minimum. Artificially increasing the measurement noise is analogous to "smoothing" trace $\left(\Sigma_{t+1}\right)$ which is a common concept in relaxation methods for findind the global minimum of a function.

In order to find $\theta_{\min }$ we require

$$
\frac{\partial\left(\operatorname{trace}\left(\Sigma_{t+1}\right)\right)}{\partial \theta}=\sum_{i=1}^{n} \frac{\xi_{i}\left[\left(\sigma_{x x}^{\prime 2}\right)_{i} \sin 2 \theta_{i}^{\prime}-\left(\sigma_{y y}^{\prime 2}\right)_{i} \sin 2 \theta_{i}^{\prime}\right]}{\left[\left(\sigma_{x x}^{\prime 2}\right)_{i} \sin ^{2} \theta_{i}^{\prime}+\left(\sigma_{y y}^{\prime 2}\right)_{i} \cos ^{2} \theta_{i}^{\prime}+\left(R_{i}\right)_{t}\right]^{2}}=0
$$

$\left(R_{i}\right)_{t}$ is artificially increased so that $\left(R_{i}\right)_{t} \gg\left(\sigma_{x x}^{\prime 2}\right)_{i},\left(\sigma_{y y}^{\prime 2}\right)_{i}$. Equation $(7)$ can then be approximated by

$$
\sum_{i=1}^{n} \frac{\xi_{i}\left[\left(\sigma_{x x}^{\prime 2}\right)_{i}-\left(\sigma_{y y}^{\prime 2}\right)_{i}\right] \sin 2 \theta_{i}^{\prime}}{\left[\frac{1}{2}\left(\left(\sigma_{x x}^{\prime 2}\right)_{i}+\left(\sigma_{y y}^{\prime 2}\right)_{i}\right)+\left(R_{i}\right)_{t}\right]^{2}}=0
$$

Substituting $\theta_{i}^{\prime}$ by $\theta-\alpha_{i}$ we get

$$
\sum_{i=1}^{n} \frac{\xi_{i}\left[\left(\sigma_{x x}^{\prime 2}\right)_{i}-\left(\sigma_{y y}^{\prime 2}\right)_{i}\right]\left(\sin 2 \theta \cos 2 \alpha_{i}-\cos 2 \theta \sin 2 \alpha_{i}\right)}{\left[\frac{1}{2}\left(\left(\sigma_{x x}^{\prime 2}\right)_{i}+\left(\sigma_{y y}^{\prime 2}\right)_{i}\right)+\left(R_{i}\right)_{t}\right]^{2}}=0 .
$$

Let

$$
\eta_{i}=\frac{\xi_{i}\left[\left(\sigma_{x x}^{\prime 2}\right)_{i}-\left(\sigma_{y y}^{\prime 2}\right)_{i}\right]}{\left[\frac{1}{2}\left(\left(\sigma_{x x}^{\prime 2}\right)_{i}+\left(\sigma_{y y}^{\prime 2}\right)_{i}\right)+\left(R_{i}\right)_{t}\right]^{2}}
$$

from equation (3.2) we get

$$
\sin 2 \theta \sum_{i=1}^{n} \eta_{i} \cos 2 \alpha_{i}-\cos 2 \theta \sum_{i=1}^{n} \eta_{i} \sin 2 \alpha_{i}=0
$$

and thus

$$
\theta=\frac{1}{2} \arctan \frac{\sum_{i=1}^{n} \eta_{i} \sin 2 \alpha_{i}}{\sum_{i=1}^{n} \eta_{i} \cos 2 \alpha_{i}}
$$

In order to find $\theta_{\min }$ we start from the value $\theta$ found in Equation (8) and converge iteratively to the global minimum. In each step the measurement noise is decreased towards its real values and convergence achieved iteratively in the new minima. The process stops when the artificially added noise vanishes.

Figure (3.2) shows the effect of "smoothing" the trace function using different amounts of artificially added noise. One can notice that the outline of the function doesn't change drastically. Thus the value of $\theta$ corresponding to the global minimum doesn't vary much through changing the amount of artificial noise. 

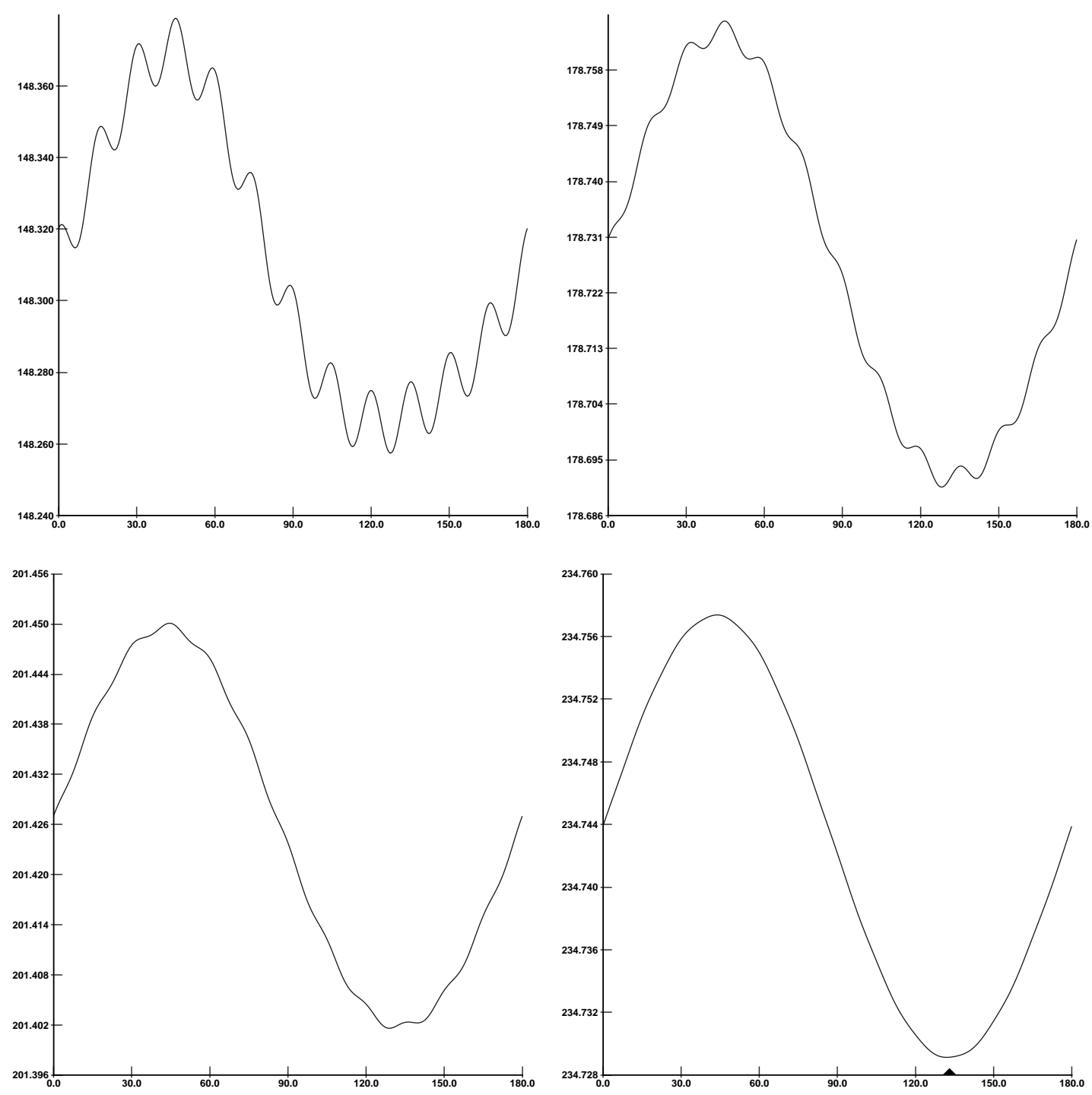

Figure 2: The values of trace $\left(\Sigma_{t+1}\right)$ ( $y$ axis) as a function of the angle $\theta$ ( $x$ axis) for 14 points with different amounts of added noise: top left $R=0.0$, top right $R=2.0$, bottom left $R=4.0$, bottom right $R=8.0$. The black triangle in the bottom right graph represents the values of $\theta$ derived from Equation (8).

\section{3D optimal positioning}

This section describes a 3D schematic generalization of the 2D ideas that were specified in the previous sections.

Let $S$ be the 3D space specified by a global right handed Cartesian coordinate system $(X, Y, Z)$. Let $F$ be a point feature in $S$. The position of $F$ is uncertain, and modelled 
as a 3 -variate Gaussian random vector

$$
P_{F}=\left(\begin{array}{c}
X_{F} \\
Y_{F} \\
Z_{F}
\end{array}\right) \sim N\left(\left(\begin{array}{c}
\hat{X}_{F} \\
\hat{Y}_{F} \\
\hat{Z}_{F}
\end{array}\right),\left(\begin{array}{ccc}
\sigma_{x x}^{2} & \sigma_{x y}^{2} & \sigma_{x z}^{2} \\
\sigma_{x y}^{2} & \sigma_{y y}^{2} & \sigma_{y z}^{2} \\
\sigma_{x z}^{2} & \sigma_{y z}^{2} & \sigma_{z z}^{2}
\end{array}\right)_{F}\right)
$$

where $\hat{P}_{F}$ is the best current estimation of the position of $F$.

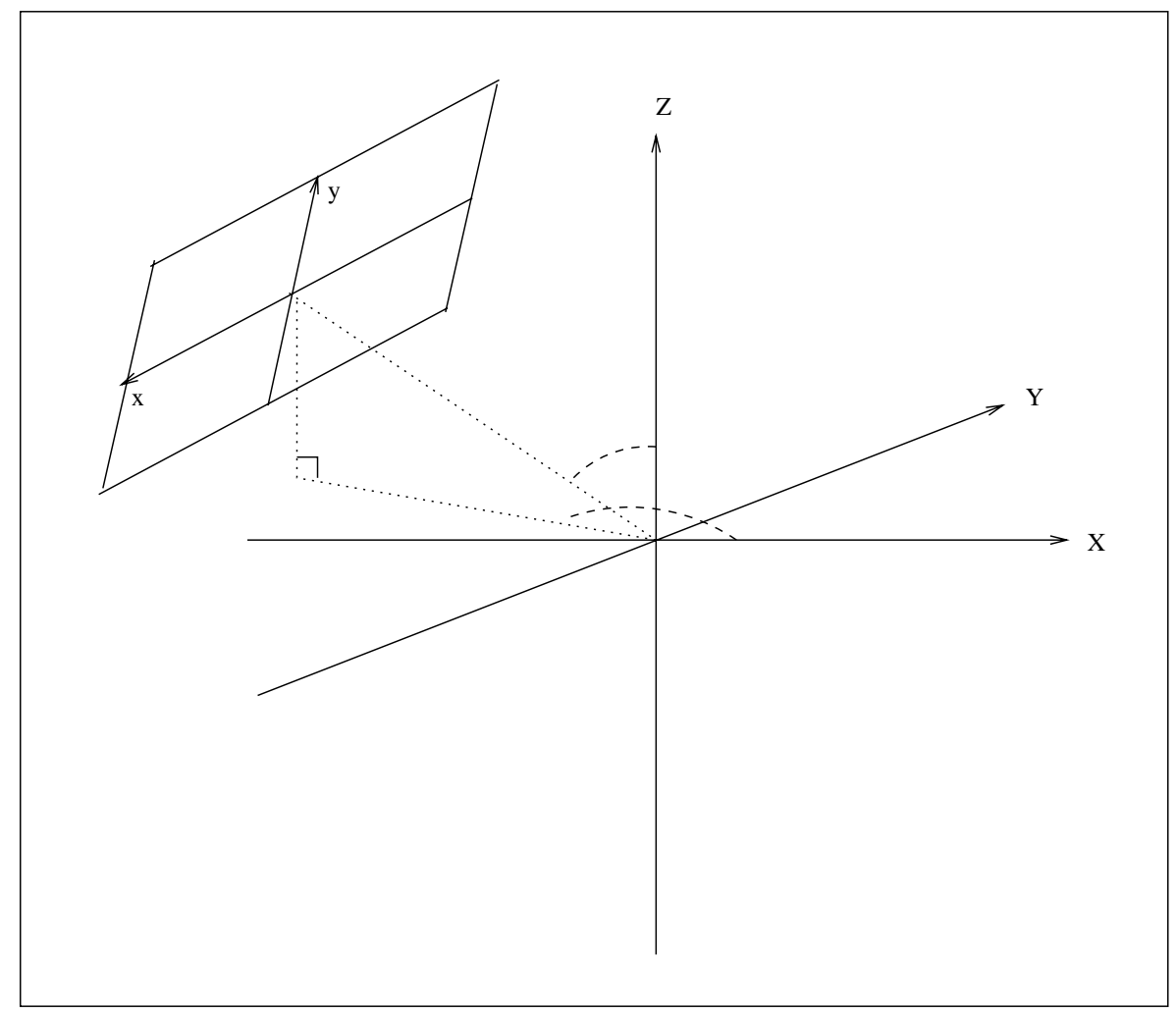

Figure 3: Coordinate systems of the 3D space and measurement projection plane

Measurements are modelled as an orthographic projection of any $P \in S$ on a plane $D$. Locations on the plane $D$ are specified by a local Cartesian coordinate system $(x, y)$. The $(x, y)$ coordinate system is positioned on $D$ such that the line that goes through the centers of the two coordinate systems is vertical to $D$. This line is specified by the pair $(\phi, \theta)$ that represents its angles relative to the $Z$ and $X$ axes accordingly. The $x$ axis of the projection plane is parallel to the space $X Y$ plane. Any measurement $(x, y)_{t}$ that takes place in time $t$ is contaminated by a bivariate zero mean Gaussian random noise $v_{t}$ whose covariance matrix is $R_{t}$. For simplicity of the analysis it is assumed that $R_{t}$ is diagonal but it could be analysed also for the general case. Then

$$
v_{t} \sim N\left(\left(\begin{array}{l}
0 \\
0
\end{array}\right),\left(\begin{array}{cc}
r_{x x}^{2} & 0 \\
0 & r_{y y}^{2}
\end{array}\right)_{t}\right) .
$$


The appropriate Gauss-Markov model is static, and its formalization is the following:

$$
\begin{gathered}
s=P_{F}=\left(\begin{array}{c}
X_{F} \\
Y_{F} \\
Z_{F}
\end{array}\right) \\
m_{t}=\left(\begin{array}{c}
x_{F} \\
y_{F}
\end{array}\right)_{t}=H_{t}^{T} s+v_{t}
\end{gathered}
$$

where

$$
H_{t}^{T}=\left(\begin{array}{clc}
-\sin \theta & \cos \theta & 0 \\
-\cos \phi \cos \theta & -\cos \phi \sin \theta & \sin \phi
\end{array}\right)
$$

Considering the case of optimizing the positioning of one feature $F$ in time $t+1$, $\operatorname{trace}\left(\Sigma_{F}\right)_{t+1}$ should be minimized. The considerations taken in the previous section concerning the rotation of the axes in order to achieve a diagonal covariance matrix hold in this case as well. Rotation of the $X Z$ plane around the $Y$ axis followed by rotation of the $X Y$ plane around the $Z$ axis results in a Gaussian which is aligned to the axes. Thus, with no loss of generality, the following analysis considers a feature $F$ whose position density function is aligned to the axes, having the covariance matrix

$$
\left(\Sigma_{F}\right)_{t}=\left(\begin{array}{ccc}
\sigma_{x x}^{2} & 0 & 0 \\
0 & \sigma_{y y}^{2} & 0 \\
0 & 0 & \sigma_{z z}^{2}
\end{array}\right)_{F}
$$

Substitution of $H_{t}^{T},\left(\Sigma_{F}\right)_{t}$ and $R_{t}$ into Equation (2) results in $\left(\Sigma_{F}\right)_{t+1}$, which is the error covariance matrix corresponding to the position of $F$ in time $t+1$. Applying the trace operator to this matrix results in an expression that represents the predicted uncertainty of the position of $F$ in time $t+1$ (see Appendix).

For many applications the assumption that the measurement noise has an identical distribution functions in both axes $\left(r_{x x}^{2}=r_{y y}^{2}\right)$ is valid. Deriving $\operatorname{trace}\left(\Sigma_{F}\right)_{t+1}$ with respect to $\theta$ and $\phi$ for this case (see Appendix) shows that the minima of the trace is obtained in two projection orientations while four other orientations are maxima and saddle points. For the particular case $\sigma_{x x}^{2}>\sigma_{y y}^{2}>\sigma_{z z}^{2}$ the characteristics of these orientations is as following:

two minima

$$
\begin{array}{ll}
\phi=0, & \theta=\text { anyvalue } \\
\phi=\pi, & \theta=\text { anyvalue }
\end{array}
$$

two maxima

$$
\begin{array}{ll}
\phi=\pi / 2 & , \quad \theta=0 \\
\phi=\pi / 2 & , \quad \theta=\pi
\end{array}
$$

and two saddle points

$$
\begin{array}{cr}
\phi=\pi / 2 & , \quad \theta=\pi / 2 \\
\phi=\pi / 2 & \quad \theta=3 \pi / 2 .
\end{array}
$$


For the cases of other orders among $\sigma_{x x}^{2}, \sigma_{y y}^{2}$ and $\sigma_{z z}^{2}$ the locations of these six points remain, while their classiffication to maxima, minima and saddle points varies. The locations of the minima are always vertical to the plane created by the two larger axes of the covariance ellipsoid.

The shape of the surface representing $\operatorname{trace}\left(\Sigma_{F}\right)_{t+1}$ as a function of $\phi$ and $\theta$ is shown in figure (4). The surface presents the particular case of a diagonal $\left(\Sigma_{F}\right)_{t}$ where $\sigma_{x x}^{2}>$ $\sigma_{y y}^{2}>\sigma_{z z}^{2}$, and $r_{x x}^{2}=r_{y y}^{2}$.

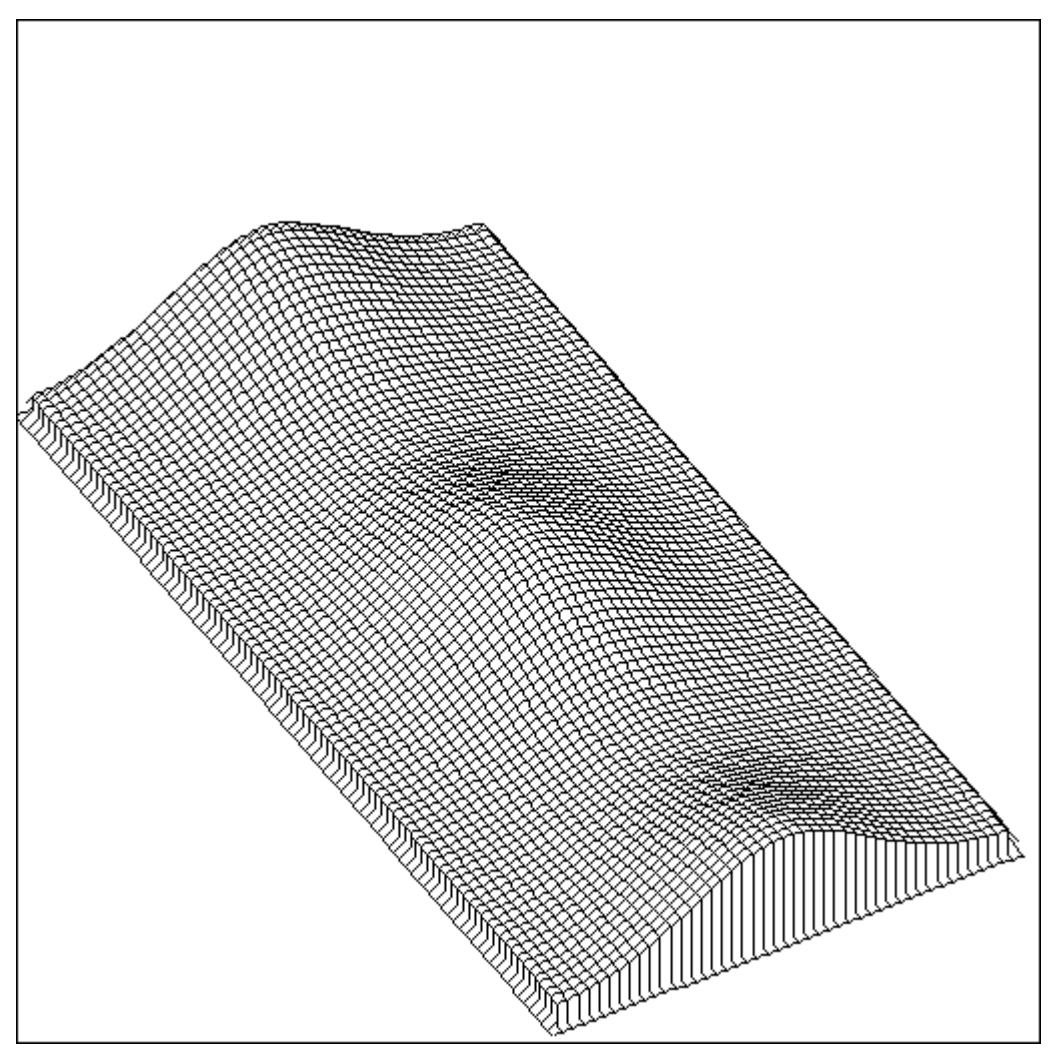

Figure 4: Surface of predicted trace $\left(\Sigma_{F}\right)_{t+1}$ as a function of the projection plane orientation

\section{$5 \quad$ Experimental Results}

The performances of the suggested active projection method were tested by simulations. The same tests were applied to the passive projection method which is currently in use. The passive approach suggests projecting in angular locations which are uniformly distributed. The number of the projections determines the angular difference between two successive projections.

Figure (5) presents the results of one of the simulations that were held. It compares the performances of the two methods for a particular case of positioning one feature with an initial uncertainty $\sigma_{x x}^{2}=100, \sigma_{y y}^{2}=20, \sigma_{x y}^{2}=0$, with noisy measurements such that 
$R=100$. The simulation contains up to 50 projections. It can be seen that the active approach is preferable. Its superiority to the passive approach is more significant as the allowed number of the projections reduces. The asymptotic behaviour of the two methods for a large number of projections is similar.

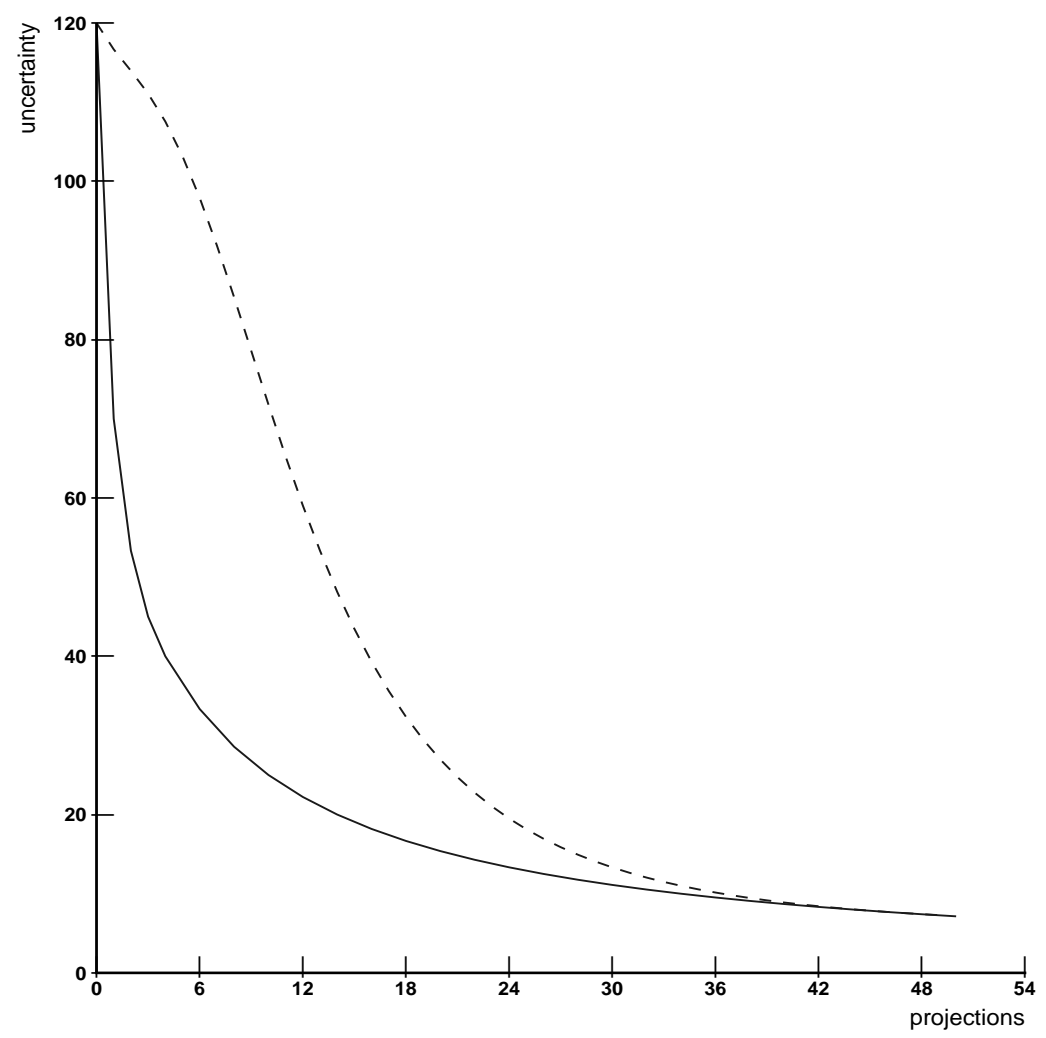

Figure 5: Results of one comparissom between the active and the passive methods. The horizontal axis presents the number of projections taken by the two methods. The vertical axis measures the uncertainty $(\operatorname{trace}(\Sigma))$ corresponding to the estimated positioning of the feature. The dashed line presents the passive method results while the solid line presents the active method results.

From Equation (4) it follows that when the angle for the next projection is chosen to be $\theta_{\min }$ then the uncertainty improvement for each active projection is

$$
\operatorname{trace}\left(\Sigma_{t}\right)-\operatorname{trace}\left(\Sigma_{t+1}\right)=\frac{\sigma_{\max }^{\prime 4}}{\sigma_{\max }^{\prime 2}+R}
$$

where $\sigma_{\max }^{\prime 2}=\max \left[\sigma_{x x}^{\prime 2}, \sigma_{y y}^{\prime 2}\right]$.

On the other hand the average uncertainty improvement for each passive projection is

$$
\frac{\sigma_{a v}^{\prime 4}}{\sigma_{a v}^{\prime 2}+R} \quad \text { where } \quad \sigma_{a v}^{\prime 2}=\frac{\sigma_{x x}^{\prime 2}+\sigma_{y y}^{\prime 2}}{2} .
$$


Thus the improvement in using the active method is more significant as $\left|\sigma_{x x}^{\prime 2}-\sigma_{y y}^{\prime 2}\right|$ increases.

\section{Conclusion}

An active approach for fine positioning of features in a noisy neighborhood has been presented. The active method is based on determination of the location of the projections according to the uncertainty corresponding to the current knowledge. The method accelerates the accuracy of the estimated features position. Its performances are better than those of the passive approach, especially where the uncertainty is nonisotropic.

A generalization of the method to polygonal models is currently in research. The number of degrees of freedom to position such models is larger than that of a unique feature point. An extension to models with uncertain shape is being considered as well.

\section{Appendix}

In section $4 s, H_{t}^{T},\left(\Sigma_{F}\right)_{t}$ and $R_{t}$ are presented for the $3 \mathrm{D}$ case. The conditional error variance associated with the estimate $\hat{s}_{t+1}$ is accepted by substituting $H_{t}^{T},\left(\Sigma_{F}\right)_{t}$ and $R_{t}$ into Equation (2) resulting in:

$$
\begin{gathered}
\operatorname{trace}\left(\Sigma_{F}\right)_{t+1}= \\
{\left[\left(\sigma_{z z}^{2} \sin ^{2} \phi+r_{y y}^{2}\right)\left(\sigma_{x x}^{2} \sin ^{2} \theta+\sigma_{y y}^{2} \cos ^{2} \theta+r_{x x}^{2}\right)+\right.} \\
\left.+\cos ^{2} \phi\left(r_{x x}^{2} \sigma_{y y}^{2} \sin ^{2} \theta+r_{x x}^{2} \sigma_{x x}^{2} \cos ^{2} \theta+\sigma_{x x}^{2} \sigma_{y y}^{2}\right)\right]^{-1} \\
{\left[r_{x x}^{2} \cos ^{2} \phi\left(\sigma_{x x}^{2} \sigma_{y y}^{2}+\sigma_{z z}^{2} \sigma_{y y}^{2} \sin ^{2} \theta+\sigma_{z z}^{2} \sigma_{x x}^{2} \cos ^{2} \theta\right)+\right.} \\
+\sigma_{z z}^{2}\left(\sigma_{x x}^{2} \sigma_{y y}^{2}+\sigma_{x x}^{2} r_{x x}^{2} \sin ^{2} \phi+\sigma_{y y}^{2} r_{x x}^{2} \sin ^{2} \phi+r_{x x}^{2} r_{y y}^{2}\right)+ \\
\left.+r_{y y}^{2}\left(\sigma_{x x}^{2} \sigma_{y y}^{2}+r_{x x}^{2} \sigma_{x x}^{2}+r_{x x}^{2} \sigma_{y y}^{2}+\sigma_{z z}^{2} \sigma_{x x}^{2} \sin ^{2} \theta+\sigma_{z z}^{2} \sigma_{y y}^{2} \cos ^{2} \theta\right)\right]
\end{gathered}
$$

Taking the partial derivatives of $\operatorname{trace}\left(\Sigma_{F}\right)_{t+1}$ with respect to $\theta$ and $\phi$ for the particular case $r_{x x}^{2}=r_{y y}^{2}=r^{2}$ shows that the minimum of the trace is obtained for the values of $\theta, \phi$ that satisfy:

$$
\begin{gathered}
\sin 2 \phi\left\{\left(r^{2}\left(\sigma_{x x}^{4}-\sigma_{y y}^{4}\right)+\sigma_{x x}^{2} \sigma_{z z}^{2}\left(\sigma_{x x}^{2}-\sigma_{z z}^{2}\right)\right)\left(r^{2}+\sigma_{y y}^{2}\right)^{2}+\right. \\
+\sin ^{2} \theta\left[r^{2}\left(\sigma_{y y}^{4}-\sigma_{x x}^{4}\right)\left(r^{4}+r^{2} \sigma_{z z}^{2}+\sigma_{z z}^{4}\right)+\right. \\
\left.\left.+\left(\sigma_{y y}^{2}-\sigma_{x x}^{2}\right)\left(r^{4} \sigma_{z z}^{4}+2 r^{2} \sigma_{x x}^{2} \sigma_{y y}^{2}\left(r^{2}-\sigma_{z z}^{2}\right)+\sigma_{x x}^{2} \sigma_{y y}^{2} \sigma_{z z}^{4}\right)\right]\right\}=0 \\
\sin 2 \theta \sin ^{2} \phi\left\{\left(r^{2}+\sigma_{z z}^{2}\right)^{2}\left(\sigma_{y y}^{2}-\sigma_{x x}^{2}\right)\left(r^{2} \sigma_{x x}^{2}+\sigma_{x x}^{2} \sigma_{y y}^{2}+r^{2} \sigma_{y y}^{2}\right)+\right. \\
\left.+r^{4} \cos ^{2} \phi\left[\sigma_{z z}^{4}\left(\sigma_{y y}^{2}-\sigma_{x x}^{2}\right)+\sigma_{y y}^{4}\left(\sigma_{x x}^{2}-\sigma_{z z}^{2}\right)+\sigma_{x x}^{4}\left(\sigma_{z z}^{2}-\sigma_{y y}^{2}\right)\right]\right\}=0
\end{gathered}
$$




\section{References}

[1] A.L. Abbot and N. Ahuja. Surface reconstruction by dynamic integration of focus, camera vergence and stereo. In Second International Conference on Computer Vision, pages, 1988.

[2] B.D.O. Anderson and J.B. Moore. Optimal Filtering. Prentice Hall, 1979.

[3] D.H. Ballard and A. Ozcandarli. Eye fixation and early vision: kinetic depth. In Second International Conference on Computer Vision, pages, 1988.

[4] J.J. Clark and N.J. Ferrier. Modal control of an attentive vision system. In Second International Conference on Computer Vision, pages, 1988.

[5] P. A. Ruymgaart and T. T. Soong. Mathematics of Kalman-Bucy Filtering. Springer, 1987. 